\title{
Finite-Element models of the human tongue: a mixed-element mesh approach
}

\author{
Pierre-Yves Rohan ${ }^{1}$, Claudio Lobos ${ }^{2}$, Mohammad Ali Nazari ${ }^{3}$, Pascal Perrier ${ }^{4}$ and Yohan Payan *5 \\ ${ }^{1}$ LBM/Institut de Biomécanique Humaine Georges Charpak, Arts et Metiers ParisTech, 151 Boulevard de \\ l'Hôpital, 75013 Paris, France \\ ${ }^{2}$ Universidad Técnica Federico Santa María, Av. Vicuña Mackenna 3939, Santiago, Chile \\ ${ }^{3}$ Mechanical Engineering Depart. Faculty of Engineering, University of Tehran, Tehran, Iran \\ ${ }^{4}$ Univ. Grenoble Alpes, GIPSA-Lab, F-38000 Grenoble, France CNRS, GIPSA-Lab, F-38000 Grenoble, \\ France \\ ${ }^{5}$ Univ. Grenoble Alpes, TIMC-IMAG, F-38000 Grenoble, France CNRS, TIMC-IMAG, F-38000 \\ Grenoble, France
}

\begin{abstract}
One of the key factors for obtaining accurate and reliable results using the Finite Element Method (FEM) is the discretization of the domain. Traditionally, two main types of elements are used for three-dimensional mesh generation: tetrahedral and hexahedral elements. Tetrahedral meshes are automatically generated but standard displacement-based tetrahedral elements generally suffer from performance issues in terms of convergence rate and accuracy of the solution associated with volumetric and shear locking. Because of these distinct disadvantages, hexahedral meshes have been used up until now for the design of biomechanical models of the orofacial system in particular for medical applications. However, hexahedral meshing is very costly and labor intensive when the mesh must be hand-made. The aim of the present contribution is to evaluate the performance of mixed-element meshes as an alternative to all-tetrahedral or all-hexahedral meshing for the analysis of problems involving nearly incompressible materials at large strains. The case study of a semi-confined compression experiment of an elastic cylindrical specimen was analyzed. The theoretical expression of deformation was derived from the literature. We observed that linear mixed-element meshes allowed results very close to those obtained using hexahedral ones. As a second experiment, we generated a mixedelement mesh of the tongue and analyze its simulated response to activation of the posterior Genioglossus muscle. Overall, our results show that mixed-element meshes can be used as computationally less demanding alternative to allhexahedral meshes for the analysis of problems involving
\end{abstract}

\footnotetext{
Permission to make digital or hard copies of all or part of this work for personal or classroom use is granted without fee provided that copies are not made or distributed for profit or commercial advantage and that copies bear this notice and the full citation on the first page. To copy otherwise, to republish, to post on servers, or to redistribute to lists requires prior specific permission and/or a fee.

PMHA-14, Aug 22-23, 2014, Vancouver, BC, CA.

Copyright remains with the author(s).
}

nearly-incompressible materials at large strains.

Keywords: Finite Element Analysis; biological soft tissue; mixed-element mesh; volumetric locking.

\section{Introduction}

Finite Element Analysis (FEA) is a very popular research tool that has been used in the last two decades to provide insights into the biomechanics of the orofacial system, and, more specifically: (i) for studying non-pathological tongue functions such as speaking [1,2,3] or swallowing [4] and (ii) for improving the clinical treatments of upper airway disorders such as obstructive sleep disorders [5, 6] and functional impairments associated with tongue surgery [7, 8]. Previous work from the authors have focused on the development of a three-dimensional (3D) finite-element (FE) biomechanical model of the main speech articulators for: (i) speech production modeling [3, 9] and (ii) the preoperative prediction of the consequences of carcinologic tongue resection on tongue mobility [8].

Finite Element meshing is a key factor for obtaining accurate and reliable results. Traditionally, two main types of elements are used for three-dimensional mesh generation: tetrahedral and hexahedral elements. Tetrahedral meshes are more easily automatically generated. However, the standard displacement-based tetrahedral element [10] suffers from several distinct disadvantages, namely (i) reduced order of convergence for strains and stresses [11] and (ii) stability issues associated to shear locking, volumetric locking $[12,13]$ and pressure checkerboard instabilities [14]. Volumetric locking in finite elements has been a major concern since its early developments. This is an artificial stiffening that appears when modeling the response of incompressible (or almost incompressible) materials such as biological soft tissues [12]. The incompressible nature of the material translates into kinematic constraints that force the Finite Elements to deform with a constant volume [15]. The consequence is that the degrees of freedom of the Finite Element mesh are no longer independent, as they should be in theory, 
resulting in an over-constrained problem.

To avoid volumetric locking associated with tetrahedral elements, hexahedral meshes have been used until now, in previous works from the authors, for the design of biomechanical models. A generic tongue mesh was manually designed by Gerard et al. [16] and improved by Buchaillard et al. [3] in the ANSYS framework, based on a combination of information extracted from the Visible Human Project ${ }^{1}$ data set and from Takemoto's (2001) dissection data [17].

In order to account for muscle force application, macrofibers, joining the FE nodes of the elements associated with a tongue muscle according to the muscle fibers' directions, were defined using external force generators. External distributed forces were enforced along the edges of the elements to simulate active muscle force generation. This modeling approach imposed important constraints on mesh design, as the hexahedral FE mesh had to be created in such a way that their vertices corresponded to the fiber directions for realistic muscle force orientation patterns. This functional modeling of muscle force distribution proved to be very useful for testing motor control models in speech production $[3,18]$ and for evaluating the consequences of oral cancer surgery on tongue mobility [8]. However, the developed tongue mesh proved to be limited: first in its capacity of handling complex articulatory patterns such as those observed in retroflex consonants (produced with the tongue tip curled back), involving strong curvature of the tongue's surface; and second in its capacity of accounting for palate/tongue contacts with sufficient spatial accuracy. Thanks to the recent progress in the development and implementation of FE formulation of muscle models, among others by the authors [9], it is now possible to decouple muscle implementation/representation and mesh design. This freedom enables to investigate crucial issues underlying mesh design such as automatic mesh generation, simulation accuracy and computation time.

An alternative to both all-tetrahedral and all-hexahedral element meshes is the use of mixed-elements (tetrahedra, triangular prisms, square pyramids, hexahedra). The overall idea is to take advantage of the benefits of combining both tetrahedral and hexahedral elements in order to automatically mesh almost any complex domain while using, thanks to a high ratio of hexahedral elements, the properties of these latter elements for numerical simulations of nearlyincompressible problems.

The goal of the present study is to evaluate the performance of a mixed-element meshing technique we recently developed [19]. The modeling context of this study is incompressible materials at large strains. Evaluation is done by comparing the simulation results obtained using the mixed-element mesh with those obtained using alltetrahedral and all-hexahedral meshes. To this end, we

\footnotetext{
1 http://www.nlm.nih.gov/research/visible/ visible_human.html
}

first evaluate the deformation of a cylindrical specimen of soft-tissues under uni-axial compression using a semiconfined configuration, for which Miller [20, 21] derived an analytical solution. Second, results provided by mixedelement tongue meshes are quantitatively compared to allhexahedral and all-tetrahedral meshes.

\section{Meshing background}

\subsection{Related work on meshing techniques}

In this section the main techniques used to generate meshes are discussed with regard to the type of elements they employ to achieve a discretization: tetrahedra, hexahedra and mixed-elements.

Automatic three-dimensional tetrahedral mesh generation has been extensively studied [22] and tetrahedral meshing procedures have become commonplace in research and industry. The versatility of tetrahedra helps to mesh complex geometries. Tetrahedral meshing procedures are mainly based on the Advancing Front Technique (AFT) [23], the Octree methods [24] and the Voronoi Delaunaybased methods [25]. Many of the developed techniques have been implemented in the preprocessing modules of commercial Finite Element software products, such as ANSYS, ABAQUS and Altair HyperMesh. They are also freely available in many open source projects, such as TetGen [26] and the Computational Geometry Algorithms Library $\left(\mathrm{CGAL}^{2}\right.$ ).

Automatic hexahedral mesh generation, on the other hand, is still considered to be a challenging research topic [27, 28]. Hexahedral meshing algorithms are generally grouped into five main categories [29, 30, 31]: (i) mapped and sweeping meshing techniques [32], (ii) medial surface techniques [33], (iii) plastering techniques [34], (iv) indirect meshing techniques and (v) grid based techniques [35]. Some of the developed techniques have been implemented in commercial FE meshing software packages such as TrueGrid which features a multi-block approach (a mapped and sweeping meshing technique). This technique has successfully been used for the creation of a hexahedral mesh of the femur [36]. However, the authors reported an extensive amount of manual intervention.

Several works, especially from the computer graphics community are contributing to help converge to a solution for the automatic all-hexahedral meshing problem. The PolyCube-Maps, introduced by Tarini et al. [37], was conceived as a mechanism for superior texture mapping in a computer graphics context. Other groups [38,39] used this technique as a starting point to develop algorithms to produce an all-hexahedral mesh. Another approach [40] needs user interaction to define skeletons that allow to produce hexahedral meshes. Finally the work of Li et al. [41] needs, first, a tetrahedral mesh to compute a singularity-restricted

\footnotetext{
2 http: / / www. cgal.org
} 
field which allows to build an all-hexahedral mesh. However the authors mention that they cannot ensure a restricted field can always be found, and, therefore, cannot ensure that a pure hexahedral mesh can always be produced with their apporoach. The main drawback of these techniques is that they tend to produce regular meshes, which may lead to an excessive number of nodes and elements in regions where they are not needed. This increases computational time for the simulation. Overall, the use of automatic hexahedral meshing procedures is still limited due to robustness and time needed to compute the simulation in some cases.

As an alternative, hybrid meshing or mixed-element methods, where tetrahedra, prisms and pyramids are combined with hexahedra, have been used in various engineering scenarios, such as, for example, Computational Fluid Dynamics applications [42] and computer graphics simulations [43]. The main issue adressed in the literature is the development of methods for interfacing tetrahedra with hexahedra [44, 45, 46].

Related work on the development of both hexahedral and mixed-element meshing algorithms reported in the literature are generally motivated by meshing challenges. Therefore, evaluation usually consists in demonstrating the performance of the developed method on several complex 3D geometries and evaluating the results with standard shapequality metrics $[47,48,29,49]$.

\subsection{Developed mixed-element meshing technique}

We recently reported a new automatic mixed-element meshing technique [50]. In a few words, our algorithm is based on the Octree technique, which starts with a bounding box (hexahedron), and recursively split it into new octants. The octants that are left completely outside the input domain are removed from the mesh. The octants intersecting the boundary of the input domain may be replaced with mixed-elements to achieve a better representation of curved domains. Finally, mixed-elements are also introduced between fine and coarse regions to produce a conformal mesh.

The main input to the algorithm developed in [50] is a closed triangular mesh describing the domain for which a volume mixed-element mesh is required. Our automatic algorithm then determines a set of octants that enclose the entire input domain. The octants can be seen as spaces that hold other smaller octants or a set of geometrical elements. In most cases the Octant is a regular hexahedron (a cube). Depending on the problem domain, more than one octant can be used as starting point: one cube may be a good starting point to enclose a brain, but a bad one to enclose a femur bone.

In this work, the Refinement Level $(R L)$ of an Octant refers to the number of recursive split into new octants. For instance, if an Octant has $R L=2$, it means that it was split into 8 new octants, and each one of them into 8 new ones, so the space covered by the original cube is now represented by $8 \times 8=64$ new octants. Note that in $2 \mathrm{D}$ this would be $4 \times 4=16$ new squares.

Now let us consider the visual example in 2D of Figure 1(a), where Octant 1 intersects a section of the boundary while the other 2 are inside the input domain. If we impose $R L=2$ for Octant 1 (see in Figure 1(b)) the resulting mesh is non-conformal. (a)

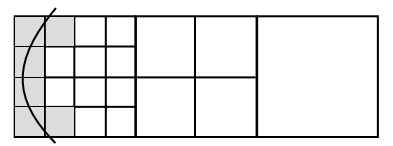

(c)

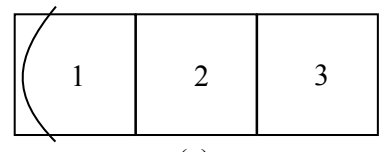

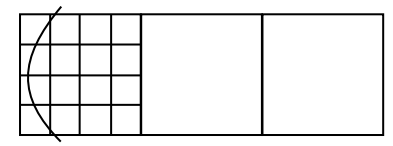

(b)

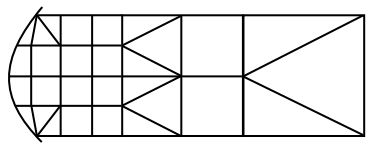

(d)
Figure 1. (a) Only octant 1 intersects the boundary, (b) Octant 1 reaches $R L=2$, (c) the mesh is one-irregular and colored small octants of Octant 1 intersects the boundary and (d) the final result after surface and transition patterns are employed to produce a conformal mesh that approximates the input boundary.

The transition between octants with different $R L$ is achieved using a set of patterns explained in [51]. Each pattern is composed by several sub-elements and consequently, the Octant is no longer one cube. This is the first step in our algorithm that introduces mixed-elements: tetrahedra, pyramids, prisms and hexahedra. We have considered all possible configurations when the difference in $R L$ between the transition Octant and its neighbors is no greater than 1. We call this property (the difference in $R L$ ): 1-irregular. For instance, in the configuration of Figure 1(b)), Octant 2 is the only one that is not 1-irregular because Octant 1 has $R L=2$ while Octant 2 has $R L=0$. In order to achieve the $1-$ irregular property we must split once Octant 2 and now the property is fulfilled throughout the mesh (see Figure 1(c)).

The next step is to decrease the chances of producing inverted elements when trying to achieve boundary representation. The colored octants in Figure 1(c) intersect the boundary, meaning that those quadrilaterals (hexahedra in 3D) have some nodes inside and outside the input domain. We will refer to them as Surface Elements or Surface octants. A previous work [50] analysed node configuration of Surface octants that may produce invalid hexahedra. If that is the case, the hexahedron is replaced by mixed-elements that decrease the chances of producing inverted elements.

The result after Surface and Transition patterns are applied can be seen in Figure 1(d). It may be seen that some unnecessary triangles were inserted in the 2D example, however this ensures the output mesh will be conformal in the much more complicated configurations of 3D problems.

As previously mentioned, the domain's boundary is not 
the only input to our algorithm. There are some options that define the way the refinement is achieved. We have defined criteria like: every Surface Octant must be refined to level $x$, or all the elements in the mesh must be refined to level $y$. These criteria can be combined. For instance the example of Figure 1 was produced demanding a $R L=2$ for Surface octants. If we now consider an example with Surface $R L=2$ and $R L=1$ for all the rest, the equivalent of Figure 1(b) would be immediately 1-irregular with octants 2 and 3 refined to the same level. The equivalent output would be the same as Figure 1(d) except for the 3 triangles representing Octant 3 , that would be represented by 4 quadrilaterals.

\section{Materials and methods}

\subsection{Analytical solution of a semi-confined compression experiment}

To assess the influence of three-dimensional finite element mesh when simulating the finite deformation of an incompressible material, the semi-confined compression experiment described in [20], for which an analytical solution has been derived based on [52], is simulated. The experiment consists in testing in compression a cylindrical specimen with low-aspect ratio. In the semi-confined configuration, the top and bottom faces of the specimen are rigidly attached to the platens of the stress-strain machine to ensure no-slip boundary conditions. Thanks to the availability of the analytical expression of the shape of the deformed specimen, it is possible to compare the theoretical maximum displacement of the cylindrical specimen with the maximum displacement obtained using the Finite Element Method. For reference, we recall the formulae in Appendix A.

\subsection{FEA of the compression experiment}

A three-dimensional Finite Element model of the semiconfined compression test was constructed using the ANSYS software. A cylindrical soft tissue specimen of 40-mm diameter and 30-mm height was modeled (Figure 2(a)). Encastre boundary conditions were imposed at the bottom and displacement boundary conditions enforced at the top surface to model the movement of the stress-strain machine platen.

Three types of meshes were tested: a coarse and fine all-tetrahedral mesh (Figure 2(b)) generated using the ANSYS meshing algorithm; a coarse and fine all-hexahedral mesh (Figure 2(c)) also generated using the ANSYS meshing algorithm; and five mixed-element meshes (Figure 2(d)) generated using the automatic mixed-element meshing algorithm developed in [19]. In this case, all the octants at the boundary of the cylinder have the same size but the discretization of the cylinder's inner section was performed differently. From left to right on figure 2, the mixed-element meshes are refined and the number of nodes increased until an almost regular hexahedral mesh is reached. For the two meshes at the bottom of figure 2(d), the octants at the boundary are smaller than the octants in the three previous meshes. Details on the different meshes are given in Table 2. In particular, the mixed-element meshes have been named according to their "refinement level" on the Surface (which are the boundary elements) and on the inside. A refinement level of 5 , for instance, means that the Octant was recursively split into 8 new octants 5 times. Accordingly, the cylinder mesh named cylS4A2, for example, means that a refinement level of 4 was used for the surface and a refinement level 2 for all the other elements.

(a)

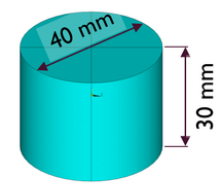

(b)
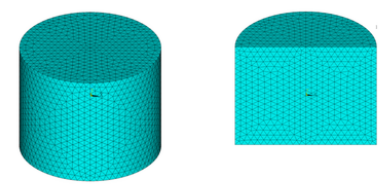

(c)
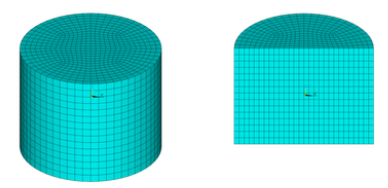

(d)
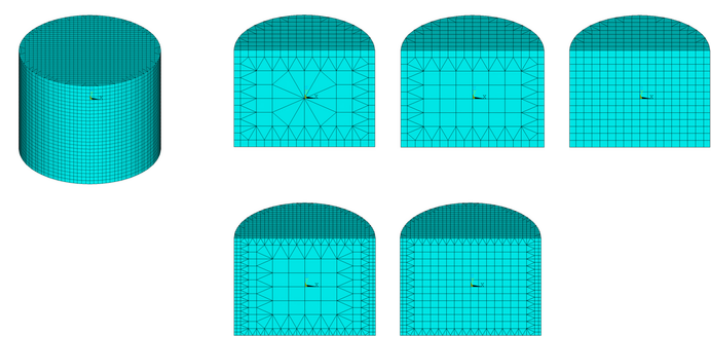

Figure 2. (a) Cylindrical specimen modeled for the FEA of the semi-confined compression test, (b) full tetrahedral mesh, (c) full hexahedral mesh and (d) mixed-element meshes.

The improvement of the performance of low-order elements, for incompressible deformations, has been, in the recent years, an important issue. Many Finite Element software products employ low-order elements because they are generally computationally less demanding and, therefore, faster. In ANSYS, two dedicated enhanced low-order elements are proposed for modeling nearly-incompressible materials: (i) a mixed u-p formulation 4-node tetrahedral element (ANSYS solid285) and (ii) a reduced-integration 8node hexahedral element with hourglass control (ANSYS solid185). The traditional displacement-based linear tetrahedral elements, which are known to perform poorly in nearincompressible regime are not available for problems involving hyperelastic materials at finite strain. In addition to these two enhanced low-order elements, two quadratic 
elements (i.e. with parabolic displacement functions) were also tested: the 10-node quadratic tetrahedral elements (ANSYS solid187) and the 20-node quadratic hexahedral element (ANSYS solid186). The influence of the number of degrees of freedom of the enhanced low-order mesh was analyzed to determine an adequate mesh size (for each element type) that provided steady model predictions without further need for an increase in mesh density. The same element size was used for both the enhanced low-order mesh and the quadratic mesh. The number of elements, nodes and degrees of freedom for each three-dimensional mesh are given in Table 2.

For the analysis, we used a Neo-Hookean hyperelastic model to describe the soft tissue specimen. Values previously reported by Buchaillard et al. [3] for the tongue were used $\left(c_{1}=1037 \mathrm{~Pa}\right.$ and $\left.K=2.10^{7} \mathrm{~Pa}\right)$.

The model was loaded by applying displacement boundary conditions on the top surface of the cylinder until it reached respectively $90 \%$ and $80 \%$ of its initial height (i.e. respectively $10 \%$ and $20 \%$ displacement). As shown by Morriss et al [21], the analytical model proposed by Miller [20] for semi-confined compression experiments of very soft tissues is limited to the range of conditions before formation of the expansion ring, i.e. before the material specimen comes in contact with the compressing platen. All the simulations were carried out with a mixed u-p formulation for all the elements.

\subsection{Parametric study}

A parametric study has been conducted to evaluate the sensitivity of the cylinder response to the ratios of hexahedra/tetrahedral elements in the mixed-element mesh. The distribution of each mesh is summarized in Table 1. The mixed-elment meshes named cylS $X \mathrm{~A} Y$ means that the cylinder has a refinement level $X$ for surface elements and a refinement level $Y$ for all the other elements.

\begin{tabular}{cccccc}
\hline Mixed-Mesh & Hex. & Pris. & Pyra. & Tet. & Total \\
cylS4A2 & $22 \%$ & $23 \%$ & $32 \%$ & $23 \%$ & 4064 \\
cylS4A3 & $25 \%$ & $24 \%$ & $30 \%$ & $21 \%$ & 3936 \\
cylS4A4 & $77 \%$ & $23 \%$ & $0 \%$ & $0 \%$ & 3864 \\
cylS5A3 & $15 \%$ & $19 \%$ & $40 \%$ & $26 \%$ & 17208 \\
cylS5A4 & $24 \%$ & $18 \%$ & $35 \%$ & $23 \%$ & 17128 \\
\hline
\end{tabular}

Table 1. Element type percentage for each mixed-element mesh. The last column shows element count.

\subsection{Post-processing}

The height of the deformed cylindrical specimen obtained from the FE simulation is normalized to allow comparison with the theoretical results given in [20]. The normalization is performed as follows: (i) the elevation $Z$ of each node on the side of the specimen is scaled relative to the initial height $H$ in order to bring all the values in the range $[-1,1]$ (ii) the current radius $r$ characterizing the position of each node on the side of the sample is normalized relative to the initial radius $R$.

\section{Results for cylindrical specimen and Interpretations}

\subsection{Results}

The deformed shape of the cylinder specimen corresponding to a displacement of the machine head inducing $\mathrm{h} / \mathrm{H}$ to change from 1 to 0.9 and from 1 to 0.8 respectively are given in figure 3. Both, the analytical solution and the computed numerical solutions are superimposed in the figure. Results obtained using linear (enhanced low-order) elements are depicted in Figure 3(a) while results obtained using quadratic elements are shown in Figure 3(b). Table 2 contains the theoretical and numerical results of the compression experiment. Quantitative evaluation of the deformed shape is based on the maximum deflection of the side of the cylinder specimen (in $Z / H=0$ ). This point also corresponds to the maximum discrepancy observed between the analytical solution and the numerical results. Table 3 contains the numerical results of the compression experiment obtained using the different mixed-element cylinder meshes.

On these two graphs, we can see that the results obtained using the dedicated ANSYS linear enhanced elements and those obtained using the quadratic elements are very close, showing that the models have converged and that increasing the number of degrees of freedom doesn't change the solution. As detailed in Table 2, increasing the number of degrees of freedom from 19206 to 483185 for the tetrahedral mesh has virtually no effect on the simulation results.

On these two graphs, we can also observe a significant difference between the numerical results (at convergence) and the analytical solution for the cylinder compression. This is due to the fact that many assumptions were made in [20] to derive the analytical solution: the theoretical solution reported is only valid for isotropic, incompressible materials and for moderate deformations when it can be assumed that planes initially perpendicular to the direction of applied extension remain plane. The material used in the numerical models is not fully-incompressible and the assumption that the planes perpendicular to the direction of the applied force remain plane during the experiment is also not verified. The error is more important as the imposed displacement increases (error increases as the imposed displacement changes from $\mathrm{h} / \mathrm{H}=0.9$ to $\mathrm{h} / \mathrm{H}=0.8$ ). This is consistent with the conclusions of [21].

Although the analytical and numerical solutions do not agree, the following conclusions can still be drawn: (i) considering the solution obtained using the second order elements as a reference value, comparable accuracy can be obtained using mixed-element meshes with much less 


\begin{tabular}{|c|c|c|c|c|c|c|c|c|c|c|c|}
\hline Model & Nodes & Elem & NDOF & $\begin{array}{l}\mathrm{h} / \mathrm{H}=0.9 \\
\mathrm{r} / \mathrm{R} \max \end{array}$ & $\begin{array}{l}\% \\
\text { error }\end{array}$ & $\mathrm{CPU}(\mathrm{s})$ & $\mathrm{vF} / \mathrm{vI}$ & $\begin{array}{l}\mathrm{h} / \mathrm{H}=0.8 \\
\mathrm{r} / \mathrm{R} \max \end{array}$ & $\begin{array}{l}\% \\
\text { error }\end{array}$ & $\mathrm{CPU}(\mathrm{s})$ & $\mathrm{vF} / \mathrm{vI}$ \\
\hline \multicolumn{4}{|c|}{ Theoretical result } & \multicolumn{4}{|l|}{1.079} & \multicolumn{4}{|l|}{1.180} \\
\hline \multicolumn{12}{|c|}{ Enhanced First order elements: } \\
\hline Tet(coarse) & 5262 & 27260 & 19206 & 1.067 & 15.3 & 1527 & 0.997 & 1.147 & 18.6 & 1369 & 0.993 \\
\hline Tet(fine) & 11079 & 60677 & 39885 & 1.067 & 15.6 & 4784 & 0.997 & 1.147 & 18.7 & 4712 & 0.993 \\
\hline Hex (coarse) & 9872 & 8820 & 25914 & 1.066 & 16.5 & 532 & 0.997 & 1.144 & 20.4 & 531 & 0.994 \\
\hline Hex(fine) & 18425 & 16896 & 50853 & 1.066 & 16.8 & 1133 & 0.997 & 1.143 & 20.6 & 1235 & 0.994 \\
\hline cylS4A2 & 2591 & 4064 & 10142 & 1.066 & 16.1 & 661 & 0.997 & 1.144 & 20.0 & 693 & 0.993 \\
\hline cylS5A4 & 13105 & 19408 & 52775 & 1.066 & 16.2 & 13267 & 0.996 & 1.144 & 20.2 & 14391 & 0.993 \\
\hline \multicolumn{12}{|c|}{ Second order elements: } \\
\hline Tet(coarse) & 39223 & 27260 & 219695 & 1.066 & 16.3 & 7327 & 0.997 & 1.144 & 19.9 & 6441 & 0.993 \\
\hline Tet(fine) & 85933 & 60677 & 483185 & 1.066 & 16.7 & 27887 & 0.997 & 1.143 & 20.5 & 29222 & 0.993 \\
\hline Hex (coarse) & 38391 & 8820 & 104247 & 1.066 & 16.5 & 4583 & 0.997 & 1.144 & 20.3 & 4713 & 0.993 \\
\hline Hex(fine) & 72113 & 16896 & 203277 & 1.066 & 16.8 & 17341 & 0.997 & 1.143 & 20.8 & 20850 & 0.993 \\
\hline cylS4A2 & 11869 & 4064 & 46985 & 1.066 & 16.7 & 593 & 0.997 & 1.143 & 20.7 & 658 & 0.993 \\
\hline cylS5A4 & 58905 & 19408 & 235981 & 1.065 & 17.2 & 7494 & 0.997 & 1.142 & 21.5 & 7878 & 0.994 \\
\hline
\end{tabular}

Table 2. Theoretical results and errors rates for the numerical simulations of the compression test (the mixed-element mesh is cylS5A3).

CPU time (660 seconds for the cylS4A2 linear mixedelement mesh instead of 27887 seconds for the fine all-tetrahedral mesh for $\mathrm{h} / \mathrm{H}=0.9$ case) (ii) the linear enhanced low-order elements available in ANSYS for modeling nearly-incompressible materials (using the mixed u-P formulation for the tetrahedral element) can correctly handle incompressibility problems in the range of deformations considered in our study.

\begin{tabular}{|c|c|c|c|c|c|}
\hline Model & Nodes & Elem. & $\begin{array}{l}\text { \% error } \\
\mathrm{h} / \mathrm{H}=0.9\end{array}$ & $\begin{array}{l}\% \text { error } \\
\mathrm{h} / \mathrm{H}=0.8\end{array}$ & $\begin{array}{l}\text { CPU (s) } \\
\mathrm{h} / \mathrm{H}=0.8\end{array}$ \\
\hline
\end{tabular}

Enhanced First order mixed-element meshes:

$\begin{array}{llllll}\text { cylS4A2 } & 2591 & 4064 & 16.1 & 18.8 & 693 \\ \text { cylS4A3 } & 2609 & 3936 & 16.1 & 20.0 & 719 \\ \text { cylS4A4 } & 4035 & 3864 & 16.1 & 20.0 & 227 \\ \text { cylS5A3 } & 11054 & 18460 & 16.2 & 20.2 & 10923 \\ \text { cylS5A4 } & 13105 & 19408 & 13.7 & 20.2 & 14391\end{array}$

Second order mixed-element meshes:

\begin{tabular}{llllll} 
cylS4A2 & 11869 & 4064 & 16.7 & 20.7 & 658 \\
cylS4A3 & 11793 & 3936 & 16.7 & 20.7 & 681 \\
cylS4A4 & 15961 & 3864 & 16.7 & 20.7 & 1729 \\
cylS5A3 & 51831 & 18460 & 17.2 & 21.5 & 4811 \\
cylS5A4 & 58905 & 19408 & 17.2 & 21.5 & 7878 \\
\hline
\end{tabular}

Table 3. Cylinder response as a function of the ratio of hexahedra/tetrahedral elements in the mixed-element mesh.

\subsection{Interpretation}

The results reported show that mixed-element meshes perform quite satisfactorily in terms of accuracy and com- putation time. Thus, mixed-element meshes are good candidates for modeling incompressible materials at large strains, as it is necessary, for example, when simulating the biomechanical response of human tongue.

The CPU time needed to compute the response was the smallest for the reduced-integration linear hexahedral element with hourglass control with an accuracy equivalent to the one that is obtained with quadratic elements (532 seconds for the coarse linear all-hexahedral mesh instead of 17 341 for the fine all-hexahedral mesh for $\mathrm{h} / \mathrm{H}=0.9$ case). The computation cost (CPU time) was slightly more important with linear mixed-elements (661 seconds for the cylS4A2 linear mixed-element mesh instead of 17341 seconds for the fine all-hexahedral mesh for $\mathrm{h} / \mathrm{H}=0.9)$. However, it should be emphasized that the mixed-element mesh can be generated automatically for complex geometries such as the human tongue, contrary to hexahedral meshes which require either a manual design or an access to the techniques cited in section 2.1, which present some drawbacks such as a regular element distribution and very long computation times.

This opens new perspectives for the development of proper FE models, for which mesh generation is critical and very time consuming. In order to evaluate the performance of the developed mixed-element meshing algorithm in a more realistic context for medical applications, the automatic design of a tongue mesh is proposed and assessed in the next section.

\section{Application to the modeling of the human tongue}

\subsection{Meshing the tongue}




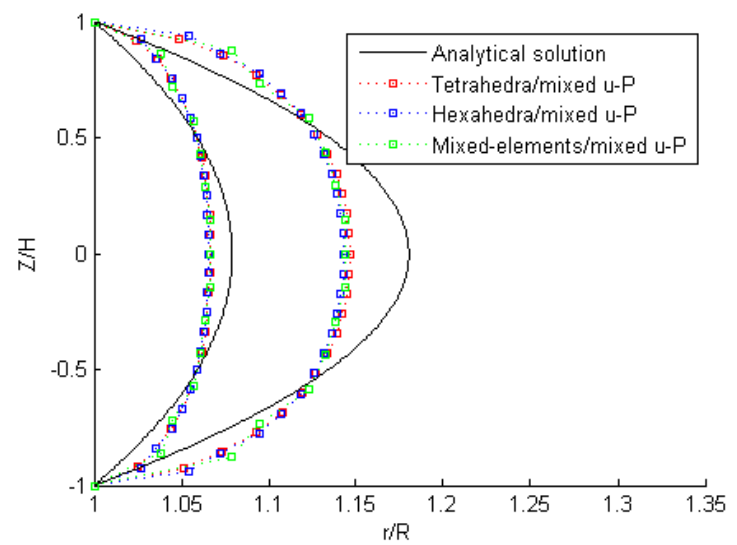

(a)

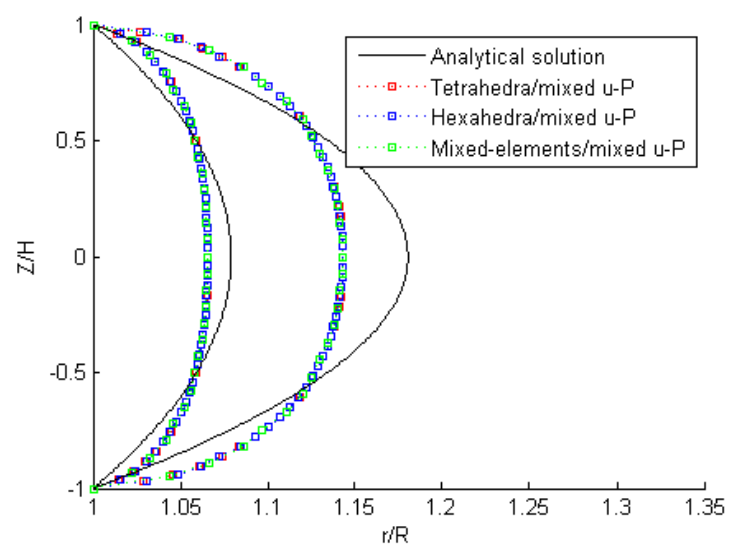

(b)

Figure 3. Plot of the theoretical and numerical results for the deformed shape of the cylinder specimen for $\mathrm{h} / \mathrm{H}=0.9$ and 0.8. (a) Numerical results computed with linear elements. (b) Numerical results computed with quadratic elements. The mixed-element mesh corresponds to cylS5A3.

As mentioned in section 1, this work is based on a generic all-hexahedral tongue mesh that was manually designed. The surface mesh of this model was extracted and used to generate new meshes. In particular, we used the surface mesh to automatically generate an all-tetrahedral mesh with ANSYS. The same surface mesh was then used to produce two different meshes using our developed automatic mixedelement mesh algorithm. All four meshes are shown in Figure 5. The two mixed-element meshes only differ in the type of refinement level used: A regular mesh with mixedelements at the surface (Figure 4(a)) on the one hand and a non-regular mesh with coarser elements in the inner part of the tongue (Figure 4(b)) on the other hand.

The main advantage of the non-regular over the regular tongue mesh is the reduction in nodes quantity. In this example, the non-regular mesh has only $75 \%$ of the number of nodes in the regular mesh. In some modeling problems computational time may be the most important constraint, therefore we include this in our analysis. By construction, in both cases, the tongue mesh consists of a high ratio of hexahedra to tetrahedra.

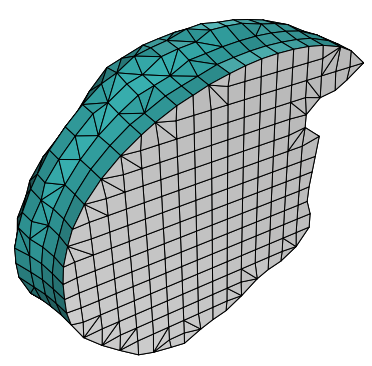

(a)

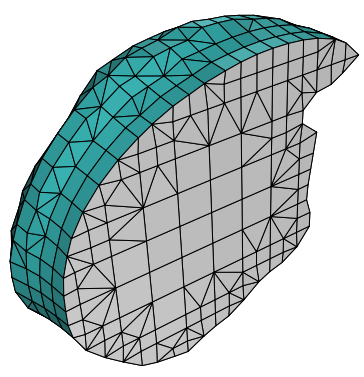

(b)
Figure 4. Using surface and transition patterns: (a) regular tongue mesh including only surface patterns, i.e., the ROI is the entire domain, (b) non-regular tongue mesh including surface and transition patterns, i.e., the ROI is limited to the set of octants intersecting the input surface.

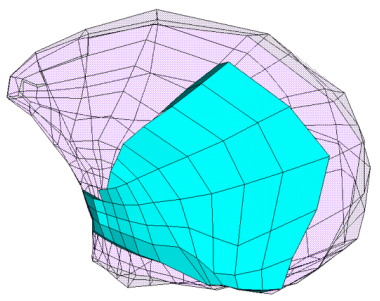

(a)

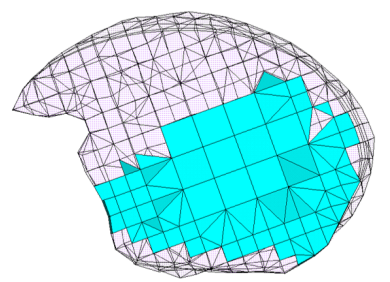

(c)

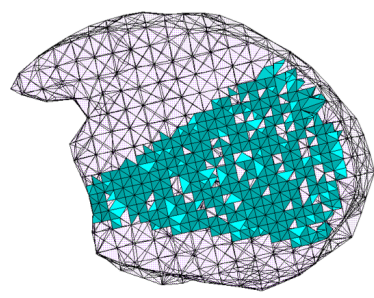

(b)

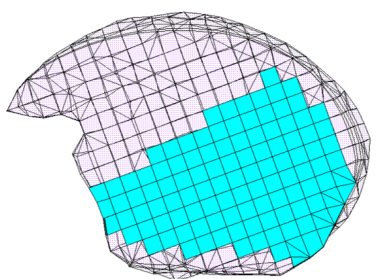

(d)
Figure 5. Tongue meshes used for the simulation of the activation of the posterior genio-glossus (GGp). (a) full hexahedral mesh, (b) full tetrahedral mesh, (c) non-regular mixedelement mesh and (d) regular mixed-element mesh.

\subsection{Biomechanical response of the tongue model to GGp activation}

The muscles influencing the tongue shape consist of the five extrinsic muscles (genioglossus, styloglossus, hyoglossus, geniohyoid and mylohyoid) and the four intrisic muscles (inferior and superior longitudinal, verticalis and transversalis) as shown in Figure 6. 


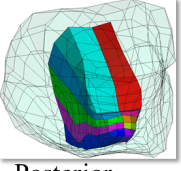

Posterior

Genioglossus

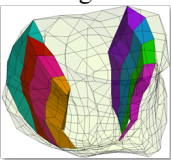

Hyoglossus

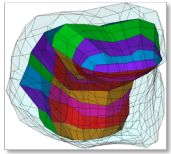

Transversalis

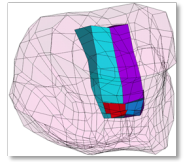

Medial

Genioglossus

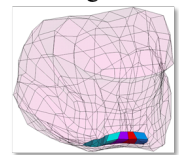

Geniohyoid

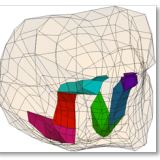

Inferior

Longitudinal

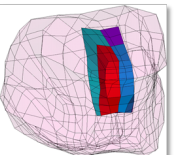

Anterior

Genioglossus

Mylohyoid

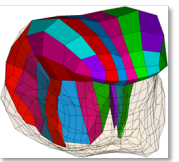

Superior

Longitudinal

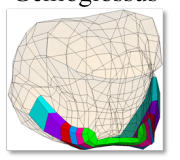

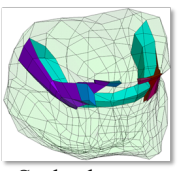

Styloglossus

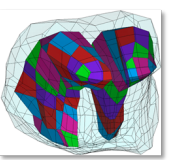

Verticalis
Figure 6 . The tongue shape is determined by five extrinsic muscles and four intrinsic muscles.

To illustrate the influence of the choice of the Finite Element Mesh on the static mechanical response of the tongue to muscle activation, we focus on the activation of the posterior genioglossus (GGp) with the four tongue meshes: a full hexahedral mesh (Figure 5(a)); a full tetrahedral mesh (Figure 5(b)) and two mixed-element meshes, a non-regular one (Figure 5(c)) and a regular one (Figure 5(d)). The activation of GGp compresses the tongue in the lower part and generates a forward and upward movement of the tongue body due to the incompressibility of tongue tissues.

In order to evaluate the differences in tongue response obtained using the various Finite Element meshes, the displacement of the tongue surface was extracted at the nodes in the mid-sagittal plane (Figure 7(c)). In order to facilitate the comparison between the different meshes, the displacement was then interpolated on a meshgrid and reported on the undeformed configuration of the tongue (Figure 7(d)).

Moreover, the following quantities were computed : (i) the Dice Similarity Coefficient (DSC), which was used to evaluate the spatial overlap of the deformed meshes in the mid-sagittal plane and (ii) the equivalent stress and strain in each (3D) tongue mesh. Given two sets A and B, the DSC is defined as:

$$
\operatorname{DSC}(A, B)=\frac{2|A \bigcap B|}{|A|+|B|}
$$

The sets A and B were defined as the surface of the polygon specified by the vertices of the tongue nodes in the midsagittal planes. The polybool Matlab function was used to compute the surface in the mid-sagittal plane corresponding to the intersection $|A \cap B|$ and the polyarea Matlab function to compute the surface area of $|A \bigcap B|,|A|$ and $|B|$. The results are summarized in Table 4.

Our results show that, as it was the case for the cylinder compression experiment, the computed displacement field

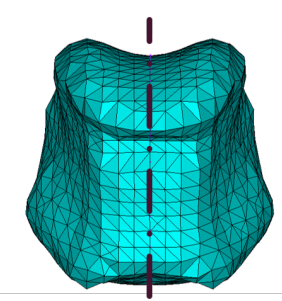

(a)

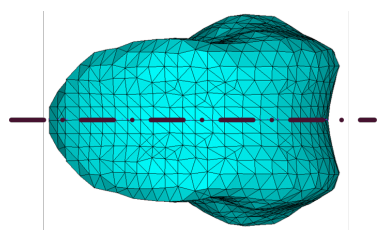

(b)

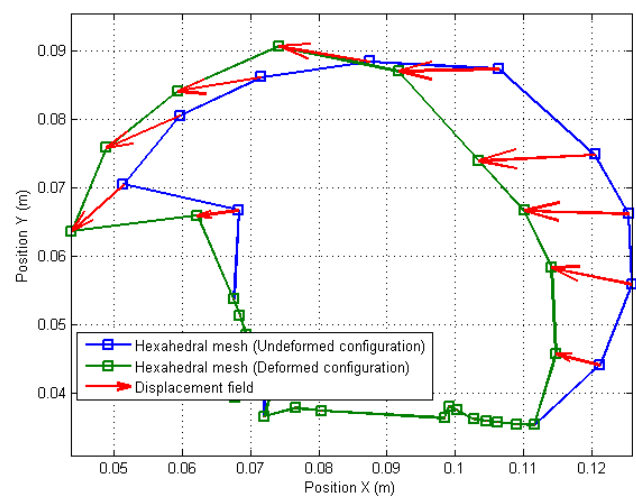

(c)

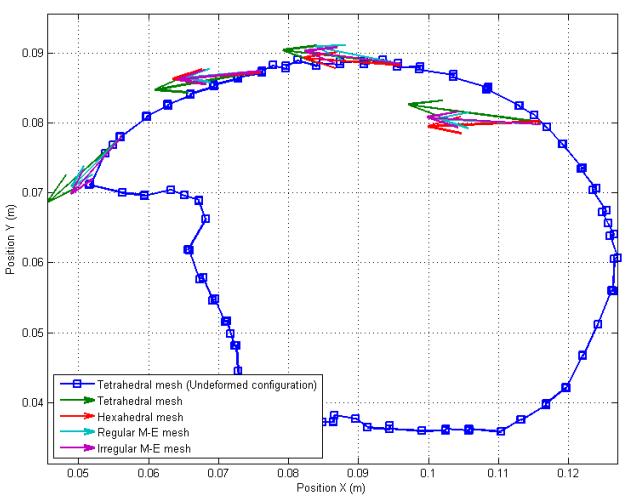

(d)
Figure 7. Results were extracted at the nodes in the midsagittal plane. (a) Mid-sagittal plane in the front view (purple) (b) Mid-sagittal plane in the top view (c) Displacement vector field at the nodes on the tongue surface in the midsagittal plane. Blue: undeformed configuration of linear hexahedral mesh; green: deformed configuration; red: displacement vector extracted at each node. (d) Displacement vector field of the tongue meshes interpolated on a common meshgrid to facilitate the comparison.

(range 15.8 - $21.6 \mathrm{~mm}$ ), equivalent stress field (range 17.8 $22.1 \mathrm{kPa}$ ) and strain field (range $20.8-26 \%$ ) are very close. Figure $7(d)$ also shows that the results predicted with the hexahedral and the regular mixed-element meshes not only match in displacement magnitudes but also in displacement 
directions. The differences in the results obtained can be explained by the mesh discretization which was relatively coarse for the manually-designed all-hexahedral mesh and fine for the all-tetrahedra and mixed-element meshes. The mean value of the DSC is equal to 0.945 with a standarddeviation of 0.02 for the deformed tongue, suggesting a high similarity between the deformed configurations.

\begin{tabular}{ll}
\hline Dice Similarity coefficient & \\
\hline Tet. and Hex. mesh & 0.939 \\
Tet. and regular M-E mesh & 0.926 \\
Tet. and irregular M-E mesh & 0.930 \\
Hex. and regular M-E mesh & 0.946 \\
Hex. and irregular M-E mesh & 0.946 \\
regular and irregular M-E mesh & 0.983 \\
& \\
\hline Mean displacement (mm) & \\
\hline Tetrahedral mesh & $21.6 \pm 5.0$ \\
Hexahedral mesh & $18.6 \pm 3.4$ \\
regular M-E mesh & $15.8 \pm 4.5$ \\
non-regular M-E mesh & $17.2 \pm 5.4$ \\
& \\
\hline Mean equivalent Stress $(\mathrm{kPa})$ & $1.3 \pm 1.3$ \\
\hline Tetrahedral mesh & $1.5 \pm 2.2$ \\
Hexahedral mesh & $1.7 \pm 2.4$ \\
regular M-E mesh & $1.5 \pm 2.4$ \\
non-regular M-E mesh & \\
\hline Mean equivalent Strain $(\%)$ & $26.7 \pm 18.0$ \\
\hline Tetrahedral mesh & $25.9 \pm 16.9$ \\
Hexahedral mesh & $24.3 \pm 17.6$ \\
regular M-E mesh & \\
non-regular M-E mesh & \\
\hline
\end{tabular}

Table 4. Tongue response.

In the present case for the modeling problem of the tongue, we do not have an exact analytical solution with which we can compare the numerical results computed using the various meshes. Based on the results obtained with the cylinder (section 4 above), we assume that the result obtained using the low-order hexahedral elements is sufficiently accurate to serve as a reference. In this context, our results show that, a satisfactory solution is obtained using the regular mixed-element tongue mesh. These findings reaffirm the important statement that mixed-element meshes can be used as a powerful alternative to hexahedral meshes for the analysis of problems involving nearly incompressible materials at large strains.

\section{Conclusion}

The use of Finite Element Analysis (FEA) as a simulation tool has grown exponentially in the biomechanical community in the last decades. These numerical models allow to compute approximate solutions of partial differential equations and derive stress-strain laws or displacement fields that would, otherwise, be impossible to obtain given the complex geometries and material constitutive laws of human organs. One of the key factors for accurate FEA is the mesh generation and the element performance. The performance of both hexahedral elements and tetrahedral elements have been evaluated in a certain number of studies in the literature [53, 54, 55, 56, 57]. Much effort has also been devoted to the development of non-locking tetrahedra, especially in the context of surgical simulations [58, 59, 60, 12].

The objective of this contribution was to assess whether mixed-element meshes could be used as an alternative to all-hexahedral meshes for the analysis of problems involving nearly-incompressible materials at large strains, as is the case for the human tongue, without introducing artificial stiffening in the form of volumetric locking that could compromise the accuracy of the solution. The case study of the semi-confined compression experiment of an elastic cylindrical specimen was analyzed using FEA and compared with the theoretical expression of deformation derived from the literature. Overall, we observed that linear mixed-element meshes gave results very close to those obtained using all-hexahedral meshes at lower computational time. Our results also showed that the linear enhanced loworder elements available in ANSYS for modeling nearlyincompressible materials (the linear 4-node tetrahedral element with mixed $\mathrm{u}-\mathrm{p}$ formulation and the linear 8-node hexahedral element with reduced-integration and hourglass control) can correctly handle incompressibility problems in the range of deformations considered in our study. We then assessed the behaviour of mixed-element meshes of the human tongue. Mixed-element meshes were thus proved to be efficient and reliable for biomechanical tongue modeling. Overall our study opens new perspectives for the development of FE models using mixed-element meshing algorithms.

\section{Appendix A}

For a cylinder of height $H$ and radius $R$ which is compressed to the new height $h$ while its radius changes to $r$, it is assumed: $z=g(Z)$ so the stretch ratio along the axis of the cylinder is:

$$
\lambda_{z}=\left.\frac{d g(Z)}{d Z}\right|_{Z=0}=\frac{h}{H}
$$

If we define $f(Z)$ as the extension ratio in the transversal plane so we have: $f(Z)=r / R$ and its value at center is $\lambda=$ $f(0)$. Due to incompressibility we have $\lambda_{z} \lambda^{2}=1$. According to derivation in [52], for Neo-Hookean material with strain energy density per reference volume: $W=C_{1}\left(I_{1}-3\right)$, 
we have:

$$
\begin{gathered}
\lambda_{z}=\frac{\sqrt{\lambda^{2}-1}}{\lambda^{2} \sec ^{-1}(\lambda)} \\
f(Z)=\lambda \cos \left(\frac{\sec ^{-1}(\lambda)}{H} Z\right)
\end{gathered}
$$

So having $\lambda_{z}$ we can find $\lambda$ and then we can compute the bulging $f(Z)$ as a function of $Z$.

\section{Acknowledgments}

The authors would like to thank the Ecos-Conicyt grant agency (Grant $\mathrm{n}^{\circ}$ C11-E01), the Fondecyt de Iniciación 11121601, the University Joseph Fourier and Grenoble INP via the Grenoble Innovation Research grant (AGIR) and the CAMI Labex under reference ANR-11-LABX-0004.

\section{References}

[1] R. Wilhelms-Tricarico, "Physiological modeling of speech production: methods for modeling soft-tissue articulators," The Journal of the Acoustical Society of America, vol. 97, pp. 3085-3098, May 1995.

[2] J. Dang and K. Honda, "Construction and control of a physiological articulatory model," The Journal of the Acoustical Society of America, vol. 115, pp. 853-870, Feb. 2004.

[3] S. Buchaillard, P. Perrier, and Y. Payan, "A biomechanical model of cardinal vowel production: muscle activations and the impact of gravity on tongue positioning," The Journal of the Acoustical Society of America, vol. 126, no. 4, pp. 20332051, 2009.

[4] J. Kieser, M. Farland, H. Jack, M. Farella, Y. Wang, and O. Rohrle, "The role of oral soft tissues in swallowing function: what can tongue pressure tell us?," Australian Dental Journal, vol. 59, pp. 155-161, June 2014.

[5] M. Zhao, T. Barber, P. A. Cistulli, K. Sutherland, and G. Rosengarten, "Simulation of upper airway occlusion without and with mandibular advancement in obstructive sleep apnea using fluid-structure interaction," Journal of Biomechanics, vol. 46, pp. 2586-2592, Oct. 2013.

[6] J.-P. V. Pelteret and B. D. Reddy, "Development of a computational biomechanical model of the human upper-airway soft-tissues toward simulating obstructive sleep apnea," Clinical Anatomy, vol. 27, pp. 182-200, Mar. 2014.

[7] S. Fujita, J. Dang, N. Suzuki, and K. Honda, "A computational tongue model and its clinical application," Oral Science International, vol. 4, pp. 97-109, Nov. 2007.

[8] S. Buchaillard, M. Brix, P. Perrier, and Y. Payan, "Simulations of the consequences of tongue surgery on tongue mobility: implications for speech production in post-surgery conditions," The International Journal of Medical Robotics and Computer Assisted Surgery, vol. 3, pp. 252-261, Sep 2007.
[9] M. A. Nazari, P. Perrier, and Y. Payan, "The distributed lambda $(\lambda)$ model (DLM): a 3-d, finite-element muscle model based on feldman's $\lambda$ model; assessment of orofacial gestures," Journal of speech, language, and hearing research, vol. 56, pp. S1909-1923, Dec 2013.

[10] K.-J. Bathe, Finite element procedures. Prentice Hall, 1996.

[11] D. J. Payen and K.-J. Bathe, "Improved stresses for the 4node tetrahedral element," Computers \& Structures, vol. 89, pp. 1265-1273, July 2011.

[12] G. R. Joldes, A. Wittek, and K. Miller, "Non-locking tetrahedral finite element for surgical simulation," Communications in Numerical Methods in Engineering, vol. 25, no. 7, pp. 827-836, 2009.

[13] Y. Onishi and K. Amaya, "A locking-free selective smoothed finite element method using tetrahedral and triangular elements with adaptive mesh rezoning for large deformation problems," International Journal for Numerical Methods in Engineering, vol. 99, pp. 354-371, Aug. 2014.

[14] F. M. Andrade Pires, E. A. de Souza Neto, and J. L. de la Cuesta Padilla, "An assessment of the average nodal volume formulation for the analysis of nearly incompressible solids under finite strains," Communications in Numerical Methods in Engineering, vol. 20, pp. 569-583, July 2004.

[15] J.-P. Ponthot, "Traitement unifié de la mécanique des milieux continus solides en grandes transformations par la méthode des eléments finis," 1995.

[16] J.-M. Gérard, R. Wilhelms-Tricarico, P. Perrier, and Y. Payan, "A 3D dynamical biomechanical tongue model to study speech motor control," Research Developments in Biomechanics, vol. 1, pp. 49-64, 2003.

[17] H. Takemoto, "Morphological analyses of the human tongue musculature for three-dimensional modeling," Journal of Speech Language and Hearing Research, vol. 44, p. 95, Feb. 2001.

[18] M. A. Nazari, P. Perrier, M. Chabanas, and Y. Payan, "Shaping by stiffening: a modeling study for lips," Motor Control, vol. 15, pp. 141-168, Jan. 2011.

[19] C. Lobos and E. González, "Mixed-element Octree: a meshing technique toward fast and real-time simulations in biomedical applications," International Journal for Numerical Methods in Biomedical Engineering, May 2015.

[20] K. Miller, "Method of testing very soft biological tissues in compression," Journal of Biomechanics, vol. 38, pp. 153158, Jan. 2005.

[21] L. Morriss, A. Wittek, and K. Miller, "Compression testing of very soft biological tissues using semi-confined configuration-a word of caution," Journal of Biomechanics, vol. 41, no. 1, pp. 235-238, 2008.

[22] W. C. Thacker, "A brief review of techniques for generating irregular computational grids," International Journal for Numerical Methods in Engineering, vol. 15, pp. 1335-1341, Sept. 1980.

[23] R. Löhner and P. Parikh, "Generation of three-dimensional unstructured grids by the advancing-front method," International Journal for Numerical Methods in Fluids, vol. 8, pp. 1135-1149, Oct. 1988.

[24] M. S. Shephard and M. K. Georges, "Automatic threedimensional mesh generation by the finite octree technique," International Journal for Numerical Methods in Engineering, vol. 32, pp. 709-749, Sept. 1991. 
[25] N. P. Weatherill and O. Hassan, "Efficient three-dimensional Delaunay triangulation with automatic point creation and imposed boundary constraints," International Journal for Numerical Methods in Engineering, vol. 37, pp. 2005-2039, June 1994.

[26] H. Si, "Tetgen, a delaunay-based quality tetrahedral mesh generator," ACM Trans. Math. Softw., vol. 41, pp. 11:111:36, February 2015.

[27] T. J. Tautges, T. Blacker, and S. A. Mitchell, "The Whisker Weaving Algorithm: A Connectivity-Based Method for Constructing All-Hexahedral Finite Element Meshes," International Journal for Numerical Methods in Engineering, vol. 39, pp. 3327-3349, Oct. 1996.

[28] M. Viceconti and F. Taddei, "Automatic generation of finite element meshes from computed tomography data," Critical Reviews in Biomedical Engineering, vol. 31, no. 1-2, pp. 2772, 2003.

[29] N. Kowalski, F. Ledoux, and P. Frey, "Smoothness driven frame field generation for hexahedral meshing," ComputerAided Design, 2015.

[30] J. Fernandes and P. Martins, "All-hexahedral remeshing for the finite element analysis of metal forming processes," $F i$ nite Elements in Analysis and Design, vol. 43, no. 8, pp. 666 - 679, 2007.

[31] Y. Zhang and C. Bajaj, "Adaptive and quality quadrilateral/hexahedral meshing from volumetric data," Computer Methods in Applied Mechanics and Engineering, vol. 195, no. 9Ü12, pp. 942 - 960, 2006.

[32] S. Hannaby, "A mapping method for mesh generation," Computers and Mathematics with Applications, vol. 16, no. 9, pp. $727-735,1988$.

[33] M. A. Price and C. G. Armstrong, "Hexahedral Mesh Generation by Medial Surface Subdivision: Part Ii. Solids with Flat and Concave Edges," International Journal for Numerical Methods in Engineering, vol. 40, pp. 111-136, Jan. 1997.

[34] T. D. Blacker and R. J. Meyers, "Seams and wedges in plastering: A 3-D hexahedral mesh generation algorithm," Engineering with Computers, vol. 9, pp. 83-93, June 1993.

[35] R. Schneiders, "A grid-based algorithm for the generation of hexahedral element meshes," Engineering with Computers, vol. 12, pp. 168-177, Sept. 1996.

[36] A. Schonning, B. Oommen, I. Ionescu, and T. Conway, "Hexahedral mesh development of free-formed geometry: The human femur exemplified," Computer-Aided Design, vol. 41, no. 8, pp. 566 - 572, 2009. Medical Modeling Special Issue: An Introduction.

[37] M. Tarini, K. Hormann, P. Cignoni, and C. Montani, "Polycube-maps," ACM Trans. Graph., vol. 23, pp. 853860, August 2004.

[38] J. Huang, T. Jiang, Z. Shi, Y. Tong, H. Bao, and M. Desbrun, "L1 based construction of polycube maps from complex shapes," ACM Trans. Graph., vol. 33, pp. 25:1-25:11, June 2014.

[39] M. Livesu, N. Vining, A. Sheffer, J. Gregson, and R. Scateni, "Polycut: Monotone graph-cuts for polycube base-complex construction," Transactions on Graphics (Proc. SIGGRAPH ASIA 2013), vol. 32, no. 6, 2013.

[40] H. Lin, H. Liao, and C. Deng, "Filling triangular mesh model with all-hex mesh by volume subdivision fitting," Tech. Rep.
TR ZJUCAD 2012 002, State Key Lab. of CAD\&CG, Zhejiang University, January 2012.

[41] Y. Li, Y. Liu, W. Xu, W. Wang, and B. Guo, "All-hex meshing using singularity-restricted field," ACM Trans. Graph., vol. 31, pp. 177:1-177:11, Nov. 2012.

[42] A. Khawaja and Y. Kallinderis, "Hybrid grid generation for turbomachinery and aerospace applications," International Journal for Numerical Methods in Engineering, vol. 49, pp. 145-166, Sept. 2000.

[43] T. Martin, E. Cohen, and R. M. Kirby, "Mixed-element volume completion from nurbs surfaces," Computers and Graphics, vol. 36, no. 5, pp. 548 - 554, 2012. Shape Modeling International (SMI) Conference 2012.

[44] S. J. Owen and S. Saigal, "Formation of pyramid elements for hexahedra to tetrahedra transitions," Computer Methods in Applied Mechanics and Engineering, vol. 190, no. 34, pp. 4505 - 4518, 2001.

[45] P. Conti, N. Hitschfeld-Kahler, and W. Fichtner, "Omega-an octree-based mixed element grid allocator for the simulation of complex 3-d device structures," IEEE Trans. on CAD of Integrated Circuits and Systems, vol. 10, no. 10, pp. 12311241, 1991.

[46] G. Nicolas and T. Fouquet, "Adaptive mesh refinement for conformal hexahedral meshes," Finite Elements in Analysis and Design, vol. 67, no. 0, pp. 1-12, 2013.

[47] G. D. Santis, M. D. Beule, K. V. Canneyt, P. Segers, P. Verdonck, and B. Verhegghe, "Full-hexahedral structured meshing for image-based computational vascular modeling," Medical Engineering and Physics, vol. 33, no. 10, pp. 1318 - 1325, 2011.

[48] H. Wu and S. Gao, "Automatic swept volume decomposition based on sweep directions extraction for hexahedral meshing," Procedia Engineering, vol. 82, pp. 136 - 148, 2014. 23rd International Meshing Roundtable (IMR23).

[49] J. Pellerin, B. Lévy, and G. Caumon, "Toward mixedelement meshing based on restricted voronoi diagrams," Procedia Engineering, vol. 82, pp. 279 - 290, 2014. 23rd International Meshing Roundtable (IMR23).

[50] C. Lobos, "A set of mixed-elements patterns for domain boundary approximation in hexahedral meshes," Studies in health technology and informatics, vol. 184, pp. 268-272, 2013. Proceedings of MMVR20.

[51] E. González and C. Lobos, "A set of mixed-element transition patterns for adaptive 3d meshing," Tech. Rep. 2014/01, Departamento de Informática, UTFSM, October 2014.

[52] W. Klingbeil and R. Shield, "Large-deformation analyses of bonded elastic mounts," Zeitschrift für angewandte Mathematik und Physik ZAMP, vol. 17, no. 2, pp. 281-305, 1966.

[53] A. Cifuentes and A. Kalbag, "A performance study of tetrahedral and hexahedral elements in 3-d finite element structural analysis," Finite Elements in Analysis and Design, vol. 12, no. 3Ü4, pp. 313 - 318, 1992.

[54] K. Polgar, M. Viceconti, and J. J. Connor, "A comparison between automatically generated linear and parabolic tetrahedra when used to mesh a human femur," Proceedings of the Institution of Mechanical Engineers, Part H: Journal of Engineering in Medicine, vol. 215, no. 1, pp. 85-94, 2001.

[55] A. Ramos and J. Simões, "Tetrahedral versus hexahedral finite elements in numerical modelling of the proximal femur," 
Medical Engineering \& Physics, vol. 28, no. 9, pp. 916 924, 2006.

[56] X. Bourdin, X. Trosseille, P. Petit, and P. Beillas, "Comparison of tetrahedral and hexahedral meshes for organ finite element modeling: An application to kidney impact," in 20th International Technical Conference for the Enhanced Safety of Vehicles, pp. 1-10, 2007.

[57] S. C. Tadepalli, A. Erdemir, and P. R. Cavanagh, "Comparison of hexahedral and tetrahedral elements in finite element analysis of the foot and footwear," Journal of Biomechanics, vol. 44, no. 12, pp. 2337 - 2343, 2011.

[58] J. Bonet and A. J. Burton, "A simple average nodal pressure tetrahedral element for incompressible and nearly incompressible dynamic explicit applications," Communications in Numerical Methods in Engineering, vol. 14, no. 5, pp. 437-449, 1998.

[59] O. C. Zienkiewicz, J. Rojek, R. L. Taylor, and M. Pastor, "Triangles and tetrahedra in explicit dynamic codes for solids," International Journal for Numerical Methods in Engineering, vol. 43, no. 3, pp. 565-583, 1998.

[60] C. Dohrmann, M. W. Heinstein, J. Jung, S. W. Key, and W. R. Witkowski, "Node-based uniform strain elements for three-node triangular and four-node tetrahedral meshes," International Journal for Numerical Methods in Engineering, vol. 47, no. 9, pp. 1549-1568, 2000. 